\title{
Linear stability of ring systems with generalized central forces
}

\author{
M. Arribas, A. Elipe, and M. Palacios
}

\begin{abstract}
Grupo de Mecánica Espacial and Instituto Universitario de Matemáticas y Aplicaciones, Universidad de Zaragoza, Zaragoza 50009, Spain

e-mail: elipe@unizar.es
\end{abstract}

Received 4 April 2008 / Accepted 1 May 2008

\begin{abstract}
We analyze the linear stability of a system of $n$ equal mass points uniformly distributed on a circle and moving about a single massive body placed at its center. We assume that the central body makes a generalized force on the points on the ring; in particular, we assume the force is generated by a Manev's type potential. This model represents several cases, for instance, when the central body is a spheroid or a radiating source. The problem contains 3 parameters, namely, the number $n$ of bodies of the ring, the mass factor $\mu$, and the radiation or oblateness coefficient $\epsilon$. For the classical case (Newtonian forces), it has been known since the seminal work of Maxwell that the problem is unstable for $n \leq 6$. For $n \geq 7$ the problem is stable when $\mu$ is within a certain interval. In this work, we determine the region $(\epsilon, \mu)$ in which the problem is stable for several values of $n$. Unstable cases $(n \leq 6)$ may become stable for negative values of $\epsilon$.
\end{abstract}

Key words. celestial mechanics

\section{Introduction}

The so-called ring problem is a classical problem in celestial mechanics. Indeed, it was introduced by Maxwell (1859) in his famous work on the stability of Saturn's rings, which was awarded the prestigious Adam's prize for the year 1856. Since then, many other scientists have been interested in this problem, for instance, let us mention Tisserand (1889), Pendse (1935), and Wintner (1947). Essentially, the problem consists of $n$ bodies of equal masses $m$ located at the vertices of a regular $n$ gon that is rotating on its own plane about its center of mass with a constant angular velocity $\omega$. Another body of mass $m_{0}$ is placed at the center of the $n$-gon. More recently, the problem has been revisited and extended in several aspects, like the computing of periodic orbits of an infinitesimal mass attracted by the ring under Newtonian forces (Scheeres 1992; Kalvouridis 1999, 2001, 2003; Pinotsis 2005), or considering more general forces (Arribas \& Elipe 2004; Elipe et al. 2007). Arribas et al. (2007) have proved that the ring configuration was central for quasihomogeneous potentials. A very interesting question raised since the beginning of the problem is the stability of the configuration. The stability depends on the mass relation $\left(\mu=m / m_{0}\right)$ of the points on the ring with relation to the central body mass, as well as on the number $n$ of primaries. Several authors like Tisserand (1889), Pendse (1935), Willerding (1986), Scheeres \& Vinh (1991), Roberts (2000), and Vanderbei \& Koleman (2007) provide several bounds for $\mu$ to have stability. It was also proven that when the number of bodies on the ring is less than 7 , the configuration is unstable. More recently, Vanderbei (2008) has considered the stability when the central mass is an oblate massive body.

In this paper, we extend these last results by considering generalized forces and, in particular, those created by the central body. Indeed, when the central body is a spheroid or a radiation source, other potentials apart from the Newtonian one must be taken into consideration. The model we use throughout this paper is the force function among the central body and a particle of mass $m$ placed at a distance $r$ from the central body, of the type

$U_{0}=k^{2} m_{0} m\left(\frac{1}{r}+\frac{\epsilon}{r^{2}}\right)$

This quasi-homogeneous potential corresponds to the Manev's type, and it models effects like the oblateness or prolateness of the central body, stellar wind, or relativistic corrections. For details and references, see the work of Arribas et al. (2007).

Note that in contrast to the recent paper by Vanderbei (2008), our parameter $\epsilon$ may be either positive, zero, or negative, whereas Vanderbei considers his parameter positive. Besides, our stability analysis is $3 \mathrm{D}$, whereas Vanderbei only considers in-plane stability.

For the extended problem considered here, which depends on the mass factor $\mu$ and $\epsilon$, we follow a scheme similar to the one given by Scheeres \& Vinh (1991) and determine the necessary conditions for linear stability. Several cases are analyzed depending on the number of bodies on the ring. We begin with $n=7$, since for the classical problem it is the first one that may be stable. The effect of the parameter $\epsilon$ is that when negative, the stability threshold for $\mu$ increases, whereas it decreases for $\epsilon>0$. The stability domain follows the same pattern for different values of $n$. For $n=6$, which is unstable for the classical problem, we find that it remains unstable for $\epsilon \geq 0$, while it is stable in a certain domain for $\epsilon<0$.

\section{Equations of motion in a synodic barycentric frame}

Let us denote by $\boldsymbol{x}_{i},(i=0, \ldots, n)$ the position vectors of the $n+1$ bodies with respect to an inertial reference frame, and by $x_{i}=\left\|\boldsymbol{x}_{i}\right\|$ its norm. Let $\boldsymbol{x}_{i j}=\boldsymbol{x}_{j}-\boldsymbol{x}_{i}$ the mutual vector and 
its norm, $x_{i j}$, the distance between bodies $P_{i}$ and $P_{j}$. The force function of the system is

$U=k^{2} m_{0} \sum_{1 \leq j \leq n} m_{j}\left(\frac{1}{x_{j 0}}+\frac{\epsilon}{x_{j 0}^{2}}\right)+k^{2} \sum_{1 \leq i<j \leq n} \frac{m_{i} m_{j}}{x_{i j}}$,

where $k^{2}$ is the gravitation constant and the parameter $\epsilon$ may be either positive, negative, or even zero. In the latest, the force is Newtonian.

The equations of motion for the central body $P_{0}$ are

$$
\begin{aligned}
\frac{\mathrm{d}^{2} \boldsymbol{x}_{0}}{\mathrm{~d} t^{2}}=\nabla_{0} U & =\nabla_{0}\left[k^{2} \sum_{j=1}^{n} m_{j}\left(\frac{1}{x_{j 0}}+\frac{\epsilon}{x_{j 0}^{2}}\right)\right] \\
& =k^{2} \sum_{j=1}^{n} m_{j}\left(\frac{1}{x_{j 0}^{3}}+\frac{2 \epsilon}{x_{j 0}^{4}}\right) \boldsymbol{x}_{j 0} .
\end{aligned}
$$

Let us choose the central body $P_{0}$ as the origin of the reference frame; that is, we choose a barycentric frame, thus, $\ddot{x_{0}}=0$, hence

$\frac{\mathrm{d}^{2} \boldsymbol{x}_{0}}{\mathrm{~d} t^{2}}=k^{2} \sum_{j=1}^{n} m_{j}\left(\frac{1}{x_{j}^{3}}+\frac{2 \epsilon}{x_{j}^{4}}\right) \boldsymbol{x}_{j}=0$.

For the bodies on the ring, the equations of motion are

$\frac{\mathrm{d}^{2} \boldsymbol{x}_{i}}{\mathrm{~d} t^{2}}=-k^{2} m_{0}\left(\frac{1}{x_{i}^{3}}+\frac{2 \epsilon}{x_{i}^{4}}\right) \boldsymbol{x}_{i}+k^{2} \sum_{j=1, j \neq i}^{n} \frac{m_{j}}{x_{i j}^{3}} \boldsymbol{x}_{i j}, \quad 1 \leq i \leq n$.

But by virtue of (2),

$m_{i}\left(\frac{1}{x_{i}^{3}}+\frac{2 \epsilon}{x_{i}^{4}}\right) \boldsymbol{x}_{i}=-\sum_{j=1, j \neq i}^{n} m_{j}\left(\frac{1}{x_{j}^{3}}+\frac{2 \epsilon}{x_{j}^{4}}\right) \boldsymbol{x}_{j}$,

then

$$
\begin{aligned}
& \frac{\mathrm{d}^{2} \boldsymbol{x}_{i}}{\mathrm{~d} t^{2}}+k^{2}\left(m_{0}+m_{i}\right)\left(\frac{1}{x_{i}^{3}}+\frac{2 \epsilon}{x_{i}^{4}}\right) \boldsymbol{x}_{i}= \\
& k^{2} \sum_{j=1, j \neq i}^{n} m_{j}\left[\frac{1}{x_{i j}^{3}} \boldsymbol{x}_{i j}-\left(\frac{1}{x_{j}^{3}}+\frac{2 \epsilon}{x_{j}^{4}}\right) \boldsymbol{x}_{j}\right] .
\end{aligned}
$$

Let us choose the units such that $k^{2} m_{0}=1$, and since masses on the ring are equal, let us introduce a parameter $\mu=m_{j} / m_{0}$, $(j=1, \ldots, n)$, then

$\frac{\mathrm{d}^{2} \boldsymbol{x}_{i}}{\mathrm{~d} t^{2}}+(1+\mu)\left(\frac{1}{x_{i}^{3}}+\frac{2 \epsilon}{x_{i}^{4}}\right) \boldsymbol{x}_{i}=\mu \sum_{j=1, j \neq i}^{n}\left[\frac{1}{x_{i j}^{3}} \boldsymbol{x}_{i j}-\left(\frac{1}{x_{j}^{3}}+\frac{2 \epsilon}{x_{j}^{4}}\right) \boldsymbol{x}_{j}\right]$.

We may introduce a scalar function $U_{i}$ defined by

$U_{i}=(1+\mu)\left(\frac{1}{x_{i}}+\frac{\epsilon}{x_{i}^{2}}\right)+\mu \sum_{j=1, j \neq i}^{n}\left(\frac{1}{x_{i j}}-\boldsymbol{x}_{i} \cdot \boldsymbol{x}_{j}\left(\frac{1}{x_{j}^{3}}+\frac{2 \epsilon}{x_{j}^{4}}\right)\right)$,

and the equations of motion in a fixed barycentric frame (3) read $\frac{\mathrm{d}^{2} \boldsymbol{x}_{i}}{\mathrm{~d} t^{2}}=\frac{\partial U_{i}}{\partial \boldsymbol{x}_{i}}$

It has been proved in Arribas et al. (2007) that the ring configuration with quasi-homogeneous potentials is a central configuration and, in particular, of relative equilibrium $\left(\boldsymbol{x}_{i}(t)=\Omega(t) \boldsymbol{x}_{i}\left(t_{0}\right)\right)$; that is to say, the configuration is rotating with angular velocity $\tilde{\omega}$ about a vector $\boldsymbol{\Omega}$ perpendicular to the plane containing the bodies. Since this case is a particular one of the quasi-homogeneous potentials analyzed in Arribas et al. (2007), there follows that the angular velocity is

$\tilde{\omega}^{2}=1+\frac{2 \epsilon}{\alpha}+\frac{\mu}{4} \sum_{k=1}^{n-1}|\csc k \theta|=\omega^{2}+\frac{2 \epsilon}{\alpha}$,

where $\omega^{2}$ is the angular velocity corresponding to the Newtonian attraction, and $\alpha$ is the radius of the ring and $\theta=\pi / n$.

With respect to a synodic barycentric frame, by virtue of the rules of derivation with respect to a rotating frame, the equations of motion are

$\frac{\mathrm{d}^{2} \boldsymbol{x}_{i}}{\mathrm{~d} t^{2}}+2 \boldsymbol{\Omega} \times \frac{\mathrm{d} \boldsymbol{x}_{i}}{\mathrm{~d} t}+\mathbf{\Omega} \times\left(\boldsymbol{\Omega} \times \boldsymbol{x}_{i}\right)=\frac{\partial U_{i}}{\partial \boldsymbol{x}_{i}}$

Due to the geometry of the problem, it is convenient to formulate it in cylindric coordinates $\left(r_{i}, \lambda_{i}, z_{i}\right)$. In these variables, the equations of motion (5) become

$$
\left.\begin{array}{l}
\ddot{r}_{j}-r_{j}\left(\dot{\lambda}_{j}+\tilde{\omega}\right)^{2}=\frac{\partial U_{j}}{\partial r_{j}} \\
r_{j} \ddot{\lambda}_{j}+2 \dot{r}_{j}\left(\dot{\lambda}_{j}+\tilde{\omega}\right)=\frac{1}{r_{j}} \frac{\partial U_{j}}{\partial \lambda_{j}} \\
\ddot{z}_{j}=\frac{\partial U_{j}}{\partial z_{j}},
\end{array}\right\} \quad j=1, \ldots, n
$$

where the potential (4) expressed in cylindrical coordinates is

$$
\begin{aligned}
U_{j} & =(1+\mu)\left[\left(r_{j}^{2}+z_{j}^{2}\right)^{-1 / 2}+\epsilon\left(r_{j}^{2}+z_{j}^{2}\right)^{-1}\right] \\
& +\mu \sum_{k=1, k \neq j}^{n}\left[\left(r_{j}^{2}+r_{k}^{2}-2 r_{j} r_{k} \cos \left(\lambda_{j}-\lambda_{k}\right)+\left(z_{j}-z_{k}\right)^{2}\right)^{-1 / 2}\right. \\
& \left.-\left(r_{j} r_{k} \cos \left(\lambda_{j}-\lambda_{k}\right)+z_{j} z_{k}\right)\left(\left(r_{k}^{2}+z_{k}^{2}\right)^{-3 / 2}+2 \epsilon\left(r_{k}^{2}+z_{k}^{2}\right)^{-2}\right)\right] .
\end{aligned}
$$

It was proved in Arribas et al. (2007) that, when bodies $P_{j}$ are placed at the vertex of the $n$-gon, $\left(r_{j}=\alpha=1, \lambda_{j}=2 \theta j, z_{j}=0\right.$, and $\tilde{\omega}^{2}=\omega^{2}+2 \epsilon$ ), the solution is an equilibrium of the equations of motion (5).

\section{Linear stability of the ring configuration}

For the analysis of the linear stability of the equilibrium solution, we need the linearized equations of motion. Thus, we introduce the variations $\rho_{j}, \sigma_{j}, z_{j}$ of the equilibria

$r_{j}=1+\rho_{j}, \quad \lambda_{j}=2 \theta j+\sigma_{j}, \quad z_{j}=z_{j}, \quad j=1 \ldots n$,

and the variational equations of the system (6) are

$\ddot{\rho}-2 \tilde{\omega} \dot{\sigma}=\tilde{\omega}^{2} \rho+A \rho+B \sigma$

$\ddot{\sigma}+2 \tilde{\omega} \dot{\rho}=C \rho+D \sigma$

$\ddot{z}=E z$

where $\boldsymbol{\rho}=\left(\rho_{1}, \ldots, \rho_{n}\right), \boldsymbol{\sigma}=\left(\sigma_{1}, \ldots, \sigma_{n}\right), z=\left(z_{1}, \ldots, z_{n}\right)$ and matrices $A, B, C, D, E$ are made of second-order partial 
derivatives of the potential evaluated at the equilibria. Thus, these matrices are symmetric, and their elements take the values

$$
\begin{aligned}
A_{i i}= & 2(1+3 \epsilon)(1+\mu)-\frac{\mu}{8} \sum_{k \neq i}\left(\left|\csc ^{3} \theta(k-i)\right|-3|\csc \theta(k-i)|\right) \\
A_{j i}= & \mu[2 \cos 2 \theta(i-j)+6 \epsilon \cos 2 \theta(i-j) \\
& \left.\left.+\frac{1}{8}\left(\cos 2 \theta(i-j)\left|\csc ^{3} \theta(i-j)\right|+3|\csc \theta(i-j)|\right)\right)\right] \\
B_{i i}= & -\mu \sum_{k \neq i} \sin 2 \theta(k-i)\left(\frac{1}{16}\left|\csc ^{3} \theta(k-i)\right|+1+2 \epsilon\right) \\
B_{j i}= & \mu \sin 2 \theta(i-j)\left(\frac{1}{16}\left|\csc ^{3} \theta(i-j)\right|+1+2 \epsilon\right) \\
C_{i i}= & B_{i i} \\
C_{j i}= & -\mu \sin 2 \theta(i-j)\left(\frac{1}{16}\left|\csc ^{3} \theta(i-j)\right|-2-6 \epsilon\right) \\
D_{i i}= & \mu \sum_{k \neq i}((1+2 \epsilon) \cos 2 \theta(k-i) \\
& \left.+\frac{1}{16}(3+\cos 2 \theta(k-i))\left|\csc ^{3} \theta(k-i)\right|\right) \\
D_{j i}= & -\mu((1+2 \epsilon) \cos 2 \theta(i-j) \\
& \left.+\frac{1}{16}(3+\cos 2 \theta(i-j))\left|\csc ^{3} \theta(i-j)\right|\right) \\
E_{i i}= & -(1+2 \epsilon)(1+\mu)-\frac{\mu}{8} \sum_{k \neq i}\left|\csc ^{3} \theta(k-i)\right| \\
E_{j i}= & -\mu(1+2 \epsilon)+\frac{\mu}{8}\left|\csc ^{3} \theta(i-j)\right| .
\end{aligned}
$$

Note that for $\epsilon=0$ we recover the Newtonian force case, which was solved by Scheeres \& Vinh (1991), so we proceed in an analogous manner to the way followed in that work.

The last $n$ equations in system (7) corresponding to the $z$ variable are uncoupled; that is, they only contain linearly variables $z_{j}$. However the first $2 n$ equations are linear in $\rho_{j}, \sigma_{j}$, but the system is coupled in those $2 n$ variables. To uncouple the system, a transformation due to Poincaré is in order. Indeed, let us introduce the $n \times n$ complex matrix $\mathcal{F}$ with elements $\mathcal{F}_{l k}=\exp (2 \theta l k \sqrt{-1})$. Its inverse matrix is simply $\mathcal{F}^{-1}=\overline{\mathcal{F}} / n$, with $\overline{\mathcal{F}}$ its conjugate matrix.

As proven by Pendse (1935), this transformation uncouples the system (7) due to the fact that matrices $A, B, C, D$, and $E$ are periodic of period $n$ and, besides, matrices $B$ and $C$ are odd functions, whereas the remaining matrices $A, D$, and $E$ are even. With this matrix, we define the complex transformation

$\rho=\mathcal{F} \xi, \quad \sigma=\mathcal{F} \eta, \quad z=\mathcal{F} \zeta$,

and system (7) is transformed into

$$
\begin{aligned}
& \ddot{\boldsymbol{\xi}}-2 \tilde{\omega} \dot{\boldsymbol{\eta}}=\tilde{\omega}^{2} \boldsymbol{\xi}+\Lambda^{A} \boldsymbol{\xi}+\Lambda^{B} \boldsymbol{\eta}, \\
& \ddot{\boldsymbol{\eta}}+2 \tilde{\omega} \dot{\boldsymbol{\xi}}=\Lambda^{C} \boldsymbol{\xi}+\Lambda^{D} \boldsymbol{\eta}, \\
& \ddot{\boldsymbol{\zeta}}=\Lambda^{E} \zeta
\end{aligned}
$$

where $\Lambda^{X}$ is the diagonal matrix of eigenvalues of matrix $X$. Note also that the new complex variables satisfy $\bar{\xi}_{j}=\xi_{n-j}$, $\bar{\eta}_{j}=\eta_{n-j}, \bar{\zeta}_{j}=\zeta_{n-j}$, so we only deal with equations with scripts $j=1,2, \ldots,[n / 2], n$, where $[a]$ denotes the integer part of $a$.

To compute the eigenvalues, we follow the procedure given by Scheeres \& Vinh (1991) and Scheeres (1992), based on the results provided by Pendse (1935). For more details about it the reader should see the original work of Pendse. By proceeding in such way, we find

$$
\begin{aligned}
& \Lambda_{j}^{A}=2 \tilde{\omega}^{2}+\mu\left(2 J_{j}-\frac{1}{4} L_{j}\right)+2 \epsilon+3 \mu \epsilon\left(2+S_{j}^{A}\right), \\
& \Lambda_{j}^{B}=\sqrt{-1} \mu\left(J_{j}+\frac{1}{8} M_{j}+\epsilon S_{j}^{B}\right), \\
& \Lambda_{j}^{C}=\sqrt{-1} \mu\left(2 J_{j}-\frac{1}{8} M_{j}+3 \epsilon S_{j}^{B}\right), \\
& \Lambda_{j}^{D}=\mu\left(-J_{j}+\frac{1}{4} N_{j}-\epsilon\left(2+S_{j}^{A}\right)\right), \\
& \Lambda_{j}^{E}=-1-2 \epsilon-\mu\left(S_{j}+\frac{1}{12}\left(L_{j}+N_{j}\right)+\epsilon\left(2+S_{j}^{E}\right)\right),
\end{aligned}
$$

where

$$
\begin{aligned}
& L_{j}=\sum_{k=1}^{n-1} \frac{1+\sin ^{2} k \theta}{\sin ^{3} k \theta} \sin ^{2} k j \theta, \quad M_{j}=\sum_{k=1}^{n-1} \frac{\cos k \theta}{\sin ^{2} k \theta} \sin 2 k j \theta, \\
& N_{j}=\sum_{k=1}^{n-1} \frac{1+\cos ^{2} k \theta}{\sin ^{3} k \theta} \sin ^{2} k j \theta, \\
& J_{j}=\left\{\begin{array}{ll}
0, & j \neq 1 \\
n / 2, & j=1
\end{array} \quad \begin{array}{ll}
0, & j \neq n \\
n, & j=n
\end{array}\right. \\
& S_{j}^{A}=2 \sum_{k=1}^{n-1} \cos (2 \theta k) \cos (2 \theta j k)= \begin{cases}n-2 & j=1, n-1 \\
-2 & \text { otherwise }\end{cases} \\
& S_{j}^{B}=2 \sum_{k=1}^{n-1} \sin (2 \theta k) \sin (2 \theta j k)= \begin{cases}n & j=1 \\
-n & j=n-1 \\
0 & \text { otherwise }\end{cases} \\
& S_{j}^{E}=2 \sum_{k=1}^{n-1} \cos 2 \theta j k=\left\{\begin{array}{l}
2 n-2 \\
-2
\end{array} \quad \begin{array}{l}
j=n \\
\text { otherwise. }
\end{array}\right.
\end{aligned}
$$

\section{Linear stability}

The roots of the characteristic equation determine the linear stability of the system (8). In this system, the last $n$ equations, those corresponding to variable $\zeta$, only depend on $\zeta$, whereas those corresponding to $\boldsymbol{\xi}$ and $\boldsymbol{\eta}$ are coupled. As a result, we split our analysis in two parts, one for the out-of-plane motion $(\zeta)$ and the other for the on-plane motion $(\boldsymbol{\xi}$ and $\boldsymbol{\eta})$.

\subsection{Out-of-plane stability}

The vertical variations are determined by the equation:

$\ddot{\boldsymbol{\zeta}}=\Lambda^{E} \zeta=-\left[1+2 \epsilon+\mu\left(S+\frac{1}{12}(L+N)+\epsilon\left(2+S^{E}\right)\right)\right] \zeta ;$

therefore, the vertical motion is stable when

$1+2 \epsilon+\mu\left(S_{j}+\frac{1}{12}\left(L_{j}+N_{j}\right)+\epsilon\left(2+S_{j}^{E}\right)\right)>0$

for each component $(1 \leq j \leq n)$. Note that $S, L, N$ are nonnegative quantities. Depending on whether $\epsilon$ is positive or negative, we have two cases. When $\epsilon>0$, the stability condition (9) is always satisfied, hence vertical motions are linearly stable. For $\epsilon<0$ we may consider two subcases:

a) $j \neq n$ : then $S_{j}=0$ and $S_{j}^{E}=-2$. Thus, the stability condition (9) reads

$$
1+2 \epsilon+\mu \frac{1}{12}\left(L_{j}+N_{j}\right)>0,
$$


b) $j=n$ : then $S_{n}=n, L_{n}=0, N_{n}=0,2+S_{n}^{E}=2 n$, and the stability condition is

$$
(1+2 \epsilon)(1+\mu n)>0 \Longleftrightarrow 1+2 \epsilon>0 .
$$

In both cases the stability condition is satisfied for $\epsilon>-1 / 2$.

\subsection{In-plane stability}

Let us now consider the variational displacements on the plane containing the primaries. The variational Eqs. (8) for variables $\xi$ and $\eta$ are

$$
\begin{aligned}
\ddot{\boldsymbol{\xi}}-2 \tilde{\omega} \dot{\boldsymbol{\eta}}= & \left(3 \tilde{\omega}^{2}+2 \mu J-\frac{1}{4} \mu L+2 \epsilon+3 \mu \epsilon\left(2+S^{A}\right)\right) \boldsymbol{\xi} \\
& +\sqrt{-1} \mu\left(J+\frac{1}{8} M+\epsilon S^{B}\right) \boldsymbol{\eta}, \\
\ddot{\boldsymbol{\eta}}+2 \tilde{\omega} \dot{\boldsymbol{\xi}}= & \sqrt{-1} \mu\left(2 J-\frac{1}{8} M+3 \epsilon S^{B}\right) \boldsymbol{\xi} \\
& +\mu\left(-J+\frac{1}{4} N-\epsilon\left(2+S^{A}\right)\right) \boldsymbol{\eta} .
\end{aligned}
$$

N.B. If the system is written in components of the vectors, i.e., $\xi_{1}, \ldots, \xi_{n}$ and $\eta_{1}, \ldots, \eta_{n}$, we have $n$ pairs of equations in the variables $\xi_{1}, \eta_{1} ; \xi_{2}, \eta_{2} ; \ldots ; \xi_{n}, \eta_{n}$. Thus, what is valid for one pair will be valid for the others.

Let us consider a generic pair of the above-mentioned equations. Since the stability of this linear system is determined by the purely imaginary roots of its characteristic equation, let us assume that $\mathrm{i} \tilde{\omega} x$ is a root of the characteristic equation with $x \in \mathbb{R}$. Then, $x$ must be a root of the quartic equation

$x^{4}-q x^{2}+r x+s=0$,

where the coefficients $q, r, s$ (for each script $j$ ) are functions of the eigenvalues of the matrices $A, B, C, D$, and $E$ :

$$
\begin{aligned}
q= & -\left(\frac{\mu}{4 \tilde{\omega}^{2}}(N-L+4 J)-1+\frac{2 \epsilon}{\tilde{\omega}^{2}}\left(1+\mu\left(2+S^{A}\right)\right)\right) \\
r= & -\frac{\mu}{2 \tilde{\omega}^{2}}(M-4 J)+\frac{2 \mu}{\tilde{\omega}^{2}} \epsilon S^{B} \\
s= & \frac{\mu}{4 \tilde{\omega}^{2}}\left(3(N-4 J)-\frac{\mu}{4 \tilde{\omega}^{2}}((N-4 J)(L-8 J)\right. \\
& \left.\left.-\frac{1}{4}(8 J+M)(16 J-M)\right)\right) \\
& +\frac{\epsilon}{\tilde{\omega}^{4}}\left(\mu^{2}\left((-5 J+L / 4+3 N / 4)\left(2+S^{A}\right)+(5 J+M / 4) S^{B}\right)\right. \\
& \left.+\mu\left(-2 J+N / 2-3 \tilde{\omega}^{2}\left(2+S^{A}\right)\right)\right) \\
& +\frac{\epsilon^{2}}{\tilde{\omega}^{4}}\left(\mu^{2}\left(3\left(S^{B}\right)^{2}-3\left(2+S^{A}\right)^{2}\right)-2 \mu\left(2+S^{A}\right)\right) .
\end{aligned}
$$

Note that for $j=n$,

$2+S_{n}^{A}=0, S_{n}^{B}=0, S_{n}=n, L_{n}=M_{n}=N_{n}=J_{n}=0$,

and the polynomial Eq. (10) is reduced to

$x^{4}-\left(1-\frac{2 \epsilon}{\tilde{\omega}^{2}}\right) x^{2}=0$.

Their four roots, namely $0,0,+\sqrt{1-2 \epsilon / \tilde{\omega}^{2}},-\sqrt{1-2 \epsilon / \tilde{\omega}^{2}}$, are real because we recall $\tilde{\omega}^{2}=\omega^{2}+2 \epsilon$. Consequently, we only need to analyze the scripts $j=1, \ldots,[n / 2]$. For these cases, to determine the number of unique real roots of the polynomial
Eq. (10), we use Sturm's theorem. To apply it, we first construct a Sturm sequence $P_{0}(x), P_{1}(x), \ldots, P_{r}(x)$, as $P_{0}(x)=P(x)$, $P_{1}(x)=P^{\prime}(x), \ldots, P_{j}(x)=-\operatorname{rem}\left(P_{j-1}(x), P_{j-2}(x)\right)$. Let $\sigma(\xi)$ be the number of sign changes (zeroes are not counted) in the sequence $P_{0}(\xi), P_{1}(\xi), \ldots, P_{r}(\xi)$. Sturm's theorem then states that for two real numbers $a<b$, the number of unique roots in the interval $(a, b]$ is $\sigma(a)-\sigma(b)$.

The Sturm sequence is, thus,

$$
\begin{aligned}
& P_{0}(x)=x^{4}-q x^{2}+r x+s \\
& P_{1}(x)=4 x^{3}-2 q x+r \\
& P_{2}(x)=2 q x^{2}-3 r x-4 s \\
& P_{3}(x)=\left(2 q^{3}-8 q s-9 r^{2}\right) x-r\left(q^{2}+12 s\right) \\
& P_{4}(x)=4 q^{3} r^{2}-27 r^{4}+16 q^{4} s-144 q r^{2} s-128 q^{2} s^{2}+256 s^{3} .
\end{aligned}
$$

Note that $P_{4}(x)$ is precisely the discriminant $\Delta$ of the quartic (10).

For the interval $(-\infty,+\infty)$, the sequence takes the following signs

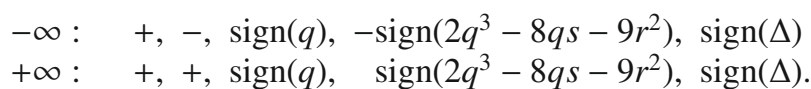

In order to have 4 real roots, it is necessary that $\sigma(+\infty)=4$ and $\sigma(-\infty)=0$; therefore, the following three conditions must be fulfilled:

$q>0$

$\Gamma=2 q\left(q^{2}-4 s\right)-9 r^{2}>0$,

$\Delta>0$.

\section{Stability domains}

\subsection{Case $n=7$}

It has been proved by several authors (Tisserand 1889; Willerding 1986; Salo \& Yoder 1988; Scheeres \& Vinh 1991; Roberts 2000; Vanderbei \& Kolemen 2007, among others), that for point-masses, if $n<7$, the system is unstable. For $n \geq 7$ these authors give some bounds for the mass factor $\mu$ to create stability.

In this problem we have two parameters, $\mu$ (the mass factor) and $\epsilon$ (the radiation or oblateness coefficient); thus, we asked ourselves whether $\epsilon$ could modify the stability and, if affirmative, how much. We begin the stability analysis with the case $n=7$, since it is the first case in which the classical problem is stable. To have stability, for given values of $\mu$ and $\epsilon$, and $n$ bodies the three conditions (11), (12), and (13) must be simultaneously fulfilled and for the scripts $j=1,2, \ldots,[n / 2]$; thus, for $n=7$, the scripts are $j=1,2,3$.

To determine the stability domain, we plot the curves $\Gamma_{j}=0$ and $\Delta_{j}=0$ for $j=1$ (left), $j=2$ (center), and $j=3$ (right) in Fig. 1. Points that satisfy the conditions $\Gamma_{j}>0$ and $\Delta_{j}>0$ are below these curves. The stability region will be the intersection of the three regions, namely, $\Gamma_{1}, \Delta_{1}>0, \Gamma_{2}, \Delta_{2}>0$ and $\Gamma_{3}, \Delta_{3}>$ 0 . This region is plotted in Fig. 2. As a matter of fact, we should check the first condition (11) for $j=1,2,3$, but this condition is less restrictive than conditions (12) and (13), since regions $q_{j}>0$ contain those with $\Gamma_{j}, \Delta_{j}>0$.

For $\epsilon=0$ a stability threshold results for the mass factor, $\mu_{0}=0.007150403074$. This bound is a little greater than 

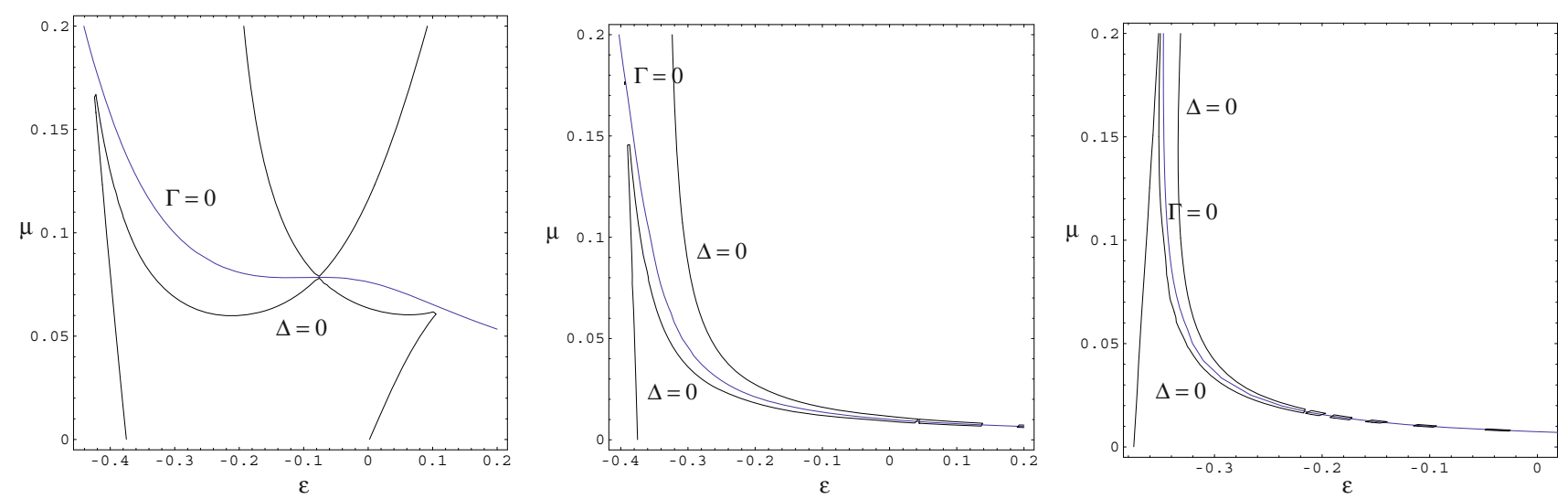

Fig. 1. Case $n=7$. Curves $\Gamma_{j}=0$ and $\Delta_{j}=0$ for $j=1$ (left), $j=2$ (center) and $j=3$ (right).
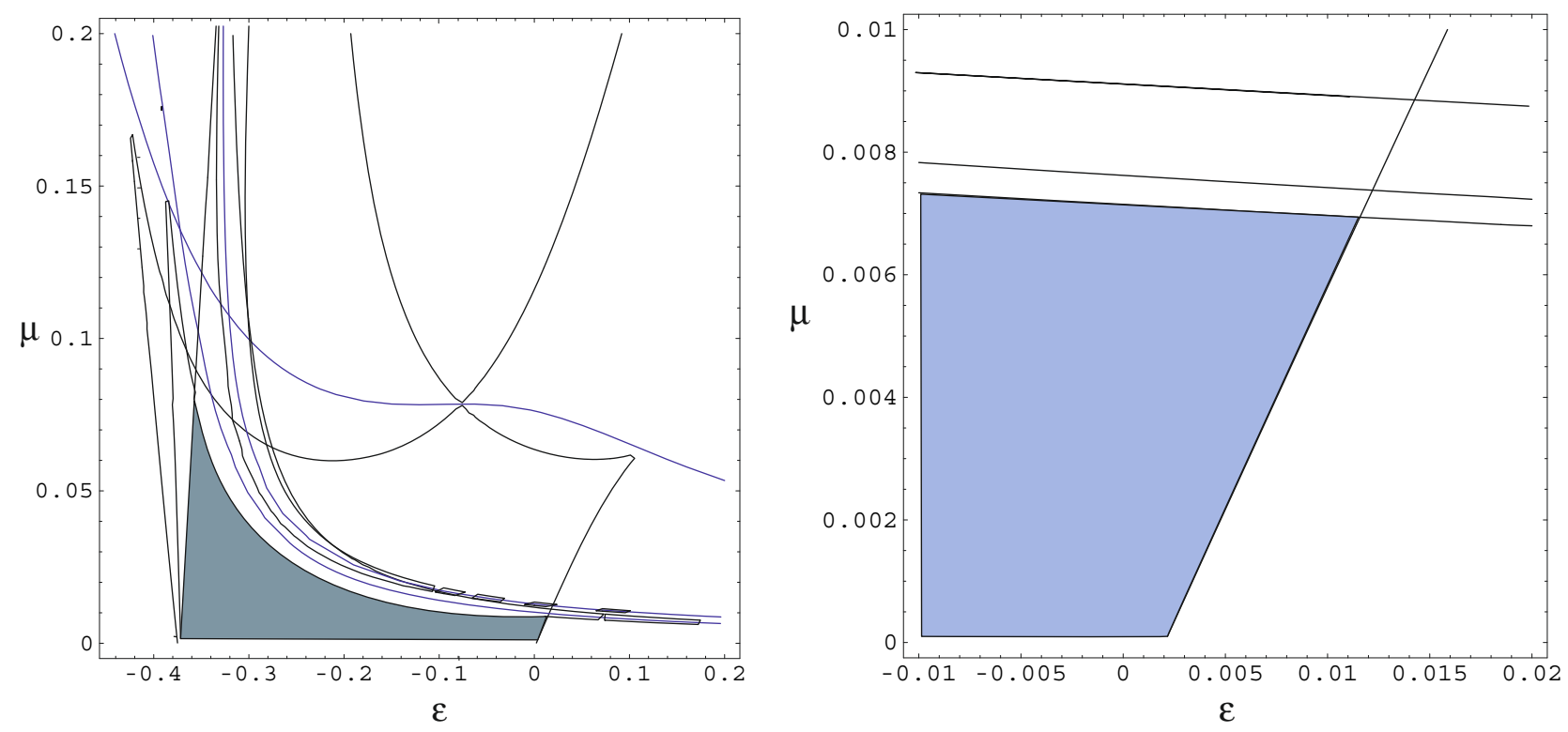

Fig. 2. Case $n=7$. The grey area corresponds to the stability region, intersection of the regions in which $\Gamma_{1}, \Delta_{1}>0, \Gamma_{2}, \Delta_{2}>0$ and $\Gamma_{3}, \Delta_{3}>0$ simultaneously. This region is inside the curves $q_{j}>0,(j=1,2,3)$. Plot on the right is a magnification of same graphics.

those obtained by several authors, e.g. Tisserand (1889) $(\mu<$ $0.00583090379)$, Roberts (2000) $(\mu<0.00670162)$, Scheeres \& Vinh $(1991)(\mu<0.007093732)$, and Vanderbei \& Kolemen (2007) $(\mu<0.00714869)$.

Besides, as can be seen from Fig. 2, the parameter $\epsilon$ contributes to the stability; thus, $\epsilon<0$ slightly increases the stability bound $\left(\mu_{-}>\mu_{0}\right)$ until it reaches a maximum at $\epsilon=-0.364556$ and, then, decreases sharply. On the other hand, for positive values of $\epsilon$ (see Fig. 2, right), the stability region is almost trapezoidal, which implies that for certain values of $\epsilon>0$, there are a lower and an upper bound of $\mu$ to have stability, that is, the configuration is stable for $\mu_{+}<\mu<\mu^{+}<\mu_{0}$. For $\epsilon$ beyond the vertex, the configuration is unstable; hence, big positive values of $\epsilon$ destroy the stability.

\subsection{Case $n \neq 7$}

As we have just seen, the parameter $\epsilon$ has an important influence on the stability; thus, our next task will be the analysis of different numbers of bodies on the ring, in particular for $n=6,8,9$, and 20 . The reason to analyze case $n=6$ is that it is the last unstable case. It would be interesting to discover if there are some values of $\epsilon$ making this configuration stable. The interest for the other cases is to see whether the stability domain follows the same pattern as for $n=7$.

By proceeding in the same way as for $n=7$, we construct Fig. 3 (left) for the case $n=6$. We had to build the regions satisfying Eqs. (11), (12), and (13) for the scripts $j=1,2,3$. From this figure, we see that the domain for stability is similar to the case $n=7$, a curvilinear trapezoid, but the configuration is only stable for some $\epsilon<0$.

For cases $n \geq 7$, the pattern is similar to the case $n=7$. We present here the cases $n=8$ and $n=20$. For $n=8$ we need to plot conditions (11), (12), and (13) for the scripts $j=$ $1,2,3,4$, whereas for $n=20$, we have to compute them for $j=$ $1, \ldots, 10$. The stability domains are represented by the shadowed areas in Fig. 3 (center and right). Essentially, the two domains look the same but distorted. In the curvilinear trapezoid, the base length increases with $\epsilon$, while the height decreases. Again, as it happened with $n=7$, we find stability for positive and negative values of $\epsilon$.

\section{Conclusions}

When generalized forces are included in the $(n+1)$-ring problem, we can model the central body as a radiation force or a spheroid. 

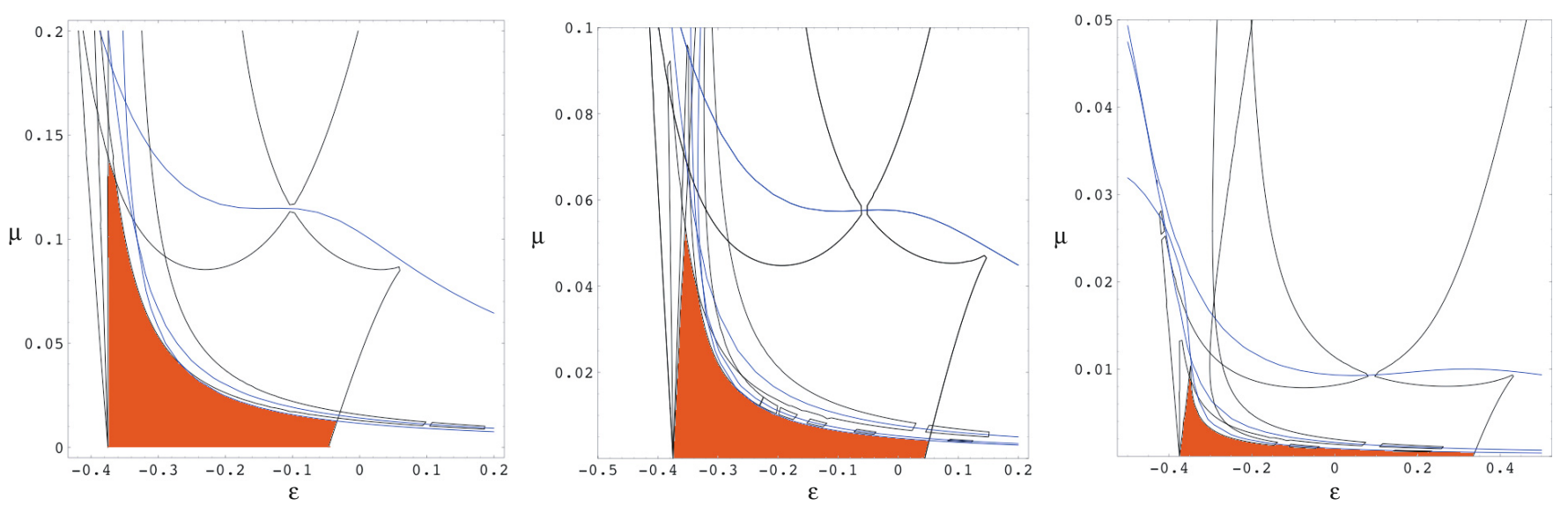

Fig. 3. Stability domains for $n=6$ (left), $n=8$ (center), and $n=20$ (right).

In this case, the problem, once the number $n$ of bodies on the ring fixed, depends on 2 parameters, $\mu$ (mass factor) and $\epsilon$ (radiation coefficient).

For this problem, we find the linear stability domain. This domain is a curvilinear trapezoid on the $\epsilon-\mu$ plane. Negative values of $\epsilon$ increases the stability bound for $\mu$, whereas positive values of $\epsilon$ reduces it. Unstable cases $(n \leq 6)$ in the classical problem become stable for negative values of $\epsilon$.

Acknowledgements. We would like to thank the anonymous referee for pointing the existence of the recent paper by Vanderbei. We acknowledge the support by the Spanish Ministry of Science and Technology (Projects \# ESP 2005-07107 and \# MTM 2006-06961).

\section{References}

Arribas, M., \& Elipe, A. 2004, Mech. Res. Comm., 31, 1

Arribas, M., Elipe, A., Kalvouridis, T., \& Palacios, M. 2007, Celest. Mech. Dyn. Astr., 99, 1
Elipe, A., Arribas, M., \& Kalvouridis, T. 2007, J. Guid. Control Dyn., 30, 1640 Kalvouridis, T., Arribas, M., \& Elipe, A. 2006, Mech. Res. Comm., 33, 811 Kalvouridis, T. J. 1999, Ap\&SS, 266, 467

Kalvouridis, T. J. 2001, Ap\&SS, 266, 579

Kalvouridis, T. J. 2003, Ap\&SS, 284, 1013

Maxwell, J. C. 1859, On the Stability of Motions of Saturn's Rings (Cambridge: Macmillan and Cia.)

Pendse, C. G. 1935, Phil. Trans. Roy. Soc., CCXXXIV-A, 145

Pinotsis, A. D. 2005, A\&A, 432, 713

Roberts, G. 2000, Monograph Series in Mathematics (World Scientific), 6, 303 Salo, H., \& Yoder, C. F. 1988, A\&A 205, 309

Scheeres, D. J. 1992, On symmetric central configurations with application to satellite motion about rings. Ph.D. Thesis, University of Michigan

Scheeres, D. J., \& Vinh, N. X. 1991, Celest. Mech. Dyn. Astr., 51, 83

Tisserand, F. 1889, Traité de Mécanique Céleste (Gauthier-Villars, Paris)

Vanderbei, R. J. 2008, J. Adv. Space Res., doi:10.1016/j.asr.2008.01.001

Vanderbei, R. J., \& Kolemen, E. 2007, AJ, 133, 656

Willerding, E. 1986, A\&A, 161, 407

Wintner, A. 1947, The Analytical Foundations of Celestial Mechanics (Princeton: Princeton University Press) 